\title{
QUASICONFORMAL EXTENSION OF HARMONIC MAPPINGS IN THE PLANE
}

\author{
Rodrigo Hernández and María J. Martín
}

\author{
Universidad Adolfo Ibáñez, Facultad de Ingeniería y Ciencias \\ Av. Padre Hurtado 750, Viña del Mar, Chile; rodrigo.hernandez@uai.cl \\ Universidad Autónoma de Madrid, Departamento de Matemáticas \\ 28049 Madrid, Spain; mariaj.martin@uam.es
}

\begin{abstract}
Let $f$ be a sense-preserving harmonic mapping in the unit disk. We give a sufficient condition in terms of the pre-Schwarzian derivative of $f$ to ensure that it can be extended to a quasiconformal map in the complex plane.
\end{abstract}

\section{Introduction}

A well-known criterion due to Becker [5] states that if a locally univalent analytic function $\phi$ in the unit disk $\mathbf{D}$ satisfies

$$
\sup _{z \in \mathbf{D}}\left|\frac{\phi^{\prime \prime}(z)}{\phi^{\prime}(z)}\right|\left(1-|z|^{2}\right) \leq 1
$$

then $\phi$ is, indeed, univalent in D. Becker and Pommerenke [6] proved later that the constant 1 is sharp.

The quotient $P \phi=\phi^{\prime \prime} / \phi^{\prime}$ is called the pre-Schwarzian derivative of $\phi$, a function that is well-defined in the unit disk for every locally univalent function $\phi$ in $\mathbf{D}$.

In that paper [5], the author also proves that if

$$
\sup _{z \in \mathbf{D}}|P \phi(z)|\left(1-|z|^{2}\right) \leq k<1,
$$

then not only $\phi$ is univalent but it has a continuous extension $\widetilde{\phi}$ to $\overline{\mathbf{D}}$ and $\widetilde{\phi}(\partial \mathbf{D})$ is a quasicircle. Indeed, using Löwner's chains, Becker shows that $\phi$ has a $K$ quasiconformal extension to the whole complex plane $\mathbf{C}$, where $K=(1+k) /(1-k)$. Moreover, Ahlfors [3] gives an explicit quasiconformal extension. Namely, the function

$$
\Phi(z)= \begin{cases}\widetilde{\phi}(z), & |z| \leq 1, \\ \phi\left(\frac{1}{\bar{z}}\right)+u\left(\frac{1}{\bar{z}}\right), & |z|>1,\end{cases}
$$

where, for $z \in \mathbf{D} \backslash\{0\}, u(z)=\phi^{\prime}(z)\left(1-|z|^{2}\right) / \bar{z}$. This mapping $\Phi$ is $K$-quasiconformal (with $K$ as above) in the complex plane whenever (2) holds and coincides with $\phi$ in D.

Let now $f$ be a locally univalent harmonic mapping defined in the unit disk and $f=h+\bar{g}$ its canonical representation, where $h$ and $g$ are analytic in $\mathbf{D}$ and $g(0)=0$. The (second) complex dilatation of $f$ is $\omega=g^{\prime} / h^{\prime}$. Since $f$ is locally univalent, by

doi:10.5186/aasfm.2013.3824

2010 Mathematics Subject Classification: Primary 30C45, 30C55.

Key words: Pre-Schwarzian derivative, quasiconformal extension, harmonic mapping.

The first author is partially supported by grants Fondecyt 1110160 and 1110321, Chile. The second author is supported by grant MTM2012-37436-C02-02, MICINN, Spain. 
Lewy's Theorem (see [12]), the Jacobian $J_{f}=\left|h^{\prime}\right|^{2}-\left|g^{\prime}\right|^{2}$ of $f$ does not vanish in the unit disk. Hence, either $J_{f}>0$ or $J_{f}<0$ in D. Any harmonic function $f$ with $J_{f}>0$ is called sense-preserving. If $J_{f}<0, f$ is said to be sense-reversing. Note that $f$ is sense-preserving if and only if $\bar{f}=g+\bar{h}$ is sense-reversing.

Throughout this paper we will consider sense-preserving harmonic mappings. It is easy to check that, in this case, $f=h+\bar{g}$ with $J_{f}=\left|h^{\prime}\right|^{2}\left(1-|\omega|^{2}\right)>0$. Equivalently, the analytic part $h$ of $f$ is locally univalent and the second complex dilatation $\omega$ is an analytic function in $\mathbf{D}$ with $\|\omega\|_{\infty}=\sup _{z \in \mathbf{D}}|\omega(z)| \leq 1$. Note that $\omega \equiv 0$ if and only if $f$ is analytic.

In a recent preprint [9], we have introduced a definition of the pre-Schwarzian derivative $P_{f}$ of locally univalent harmonic mappings $f=h+\bar{g}$ in the unit disk. If $f$ is sense-preserving, then

$$
P_{f}=\frac{h^{\prime \prime}}{h^{\prime}}-\frac{\bar{\omega} \omega^{\prime}}{\left(1-|\omega|^{2}\right)}
$$

in D. Using this definition for the pre-Schwarzian derivative of $f$, we proved (see $[9$, Theorem 9]) that if $f=h+\bar{g}$ is a sense-preserving harmonic mapping in the unit disk with second complex dilatation $\omega$ and

$$
\left|P_{f}(z)\right|\left(1-|z|^{2}\right)+\frac{\left|\omega^{\prime}(z)\right|\left(1-|z|^{2}\right)}{1-|\omega(z)|^{2}} \leq 1
$$

for all $|z|<1$, then $f$ is univalent in $\mathbf{D}$. The constant 1 is sharp. by

The second term in (3) is the modulus of the hyperbolic derivative $\omega^{*}$ of $\omega$ defined

$$
\omega^{*}(z)=\frac{\omega^{\prime}(z)\left(1-|z|^{2}\right)}{1-|\omega(z)|^{2}}, \quad z \in \mathbf{D} .
$$

As we mentioned before, if $\phi$ is analytic, then it is a sense-preserving harmonic mapping with dilatation $\omega \equiv 0$. Hence, (3) generalizes Becker's criterion of univalence (1) to sense-preserving harmonic mappings.

The purpose of this paper is to show that analogous results to those due to Becker and Ahlfors that we have mentioned before also hold for the family of sensepreserving harmonic mappings in the unit disk. Concretely, we prove the following theorems.

Theorem 1. Let $f=h+\bar{g}$ be a sense-preserving harmonic mapping in the unit disk with second complex dilatation $\omega$. Assume that

$$
\left|P_{f}(z)\left(1-|z|^{2}\right)\right|+\left|\omega^{*}(z)\right| \leq k<1, \quad z \in \mathbf{D} .
$$

Then, the harmonic mapping $f$ has a continuous and injective extension $\widetilde{f}$ to $\overline{\mathbf{D}}$. Moreover, the function

$$
F(z)= \begin{cases}\widetilde{f}(z), & |z| \leq 1 \\ f\left(\frac{1}{\bar{z}}\right)+U\left(\frac{1}{\bar{z}}\right), & |z|>1\end{cases}
$$

is a homeomorphic extension of $f$ to the whole complex plane onto itself. The function $U$ that appears in (5) is defined by

$$
U(z)=\frac{h^{\prime}(z)\left(1-|z|^{2}\right)}{\bar{z}}+\frac{\overline{g^{\prime}(z)}\left(1-|z|^{2}\right)}{z}, \quad z \in \mathbf{D} \backslash\{0\} .
$$


An extra condition on the second complex dilatation $\omega$ of the mapping $f$ is needed in the following theorem. We will justify in Section 3 below why this condition cannot be omitted.

Theorem 2. Let the sense-preserving harmonic mapping $f$ satisfy (4). If, in addition, $\|\omega\|_{\infty}<1$, then $\widetilde{f}(\partial \mathbf{D})$ is a quasicircle and $f$ can be extended to a quasiconformal map in C. Indeed, the function defined by (5) is an explicit $K$-quasiconformal extension of $f$ whenever

$$
k<\frac{1-\|\omega\|_{\infty}}{1+\|\omega\|_{\infty}} .
$$

The constant $K$ equals

$$
K=\frac{(1+k)+(1-k)\|\omega\|_{\infty}}{(1-k)-(1+k)\|\omega\|_{\infty}}
$$

\section{Background}

1.1. Quasiconformal maps. A complex valued function $f$ on a domain $\Omega \subset \overline{\mathbf{C}}$ is absolutely continuous on lines (ACL) in $\Omega$ if for every rectangle of the form $R=$ $\{x+i y: a<x<b, c<y<d\}$ with $\bar{R} \subset \Omega \backslash\left\{\infty, f^{-1}(\infty)\right\}$, the map $f$ is absolutely continuous on almost every horizontal and vertical line in $R$.

Let $K$ be a real positive number with $K \geq 1$. A sense-preserving homeomorphism $f: \Omega \rightarrow \mathbf{C}$ is called quasiconformal (or $K$-quasiconformal) if $f$ is ACL in $\Omega$ and $\left|f_{\bar{z}}\right| \leq$ $k\left|f_{z}\right|$ almost everywhere in $\Omega$, where $k=(K-1) /(K+1)$. The 1-quasiconformal mappings are the conformal mappings.

The complex dilatation of $f$ is the ratio $\mu_{f}=f_{\bar{z}} / f_{z}$. If $f$ is sense-preserving, then $0 \leq\left|\mu_{f}\right|<1$. Hence, a sense-preserving ACL homeomorphism $f$ is quasiconformal if and only if $\mu_{f}$ is bounded away from 1 in the given region. The second complex dilatation $\omega_{f}$ of $f$ is defined by $\omega_{f}=\overline{f_{\bar{z}}} / f_{z}$. Since $\left|\omega_{f}\right|=\left|\mu_{f}\right|$, we conclude that $f$ is quasiconformal if and only if $\left|\omega_{f}\right| \leq k<1$.

Let now $f$ be a harmonic mapping defined in a simply connected domain $\Omega \subset \mathbf{C}$. It is not difficult to check that $f$ has the representation (unique up to an additive constant) $f=h+\bar{g}$ for two analytic functions $h$ and $g$ in $\Omega$.

For harmonic mappings $f$ in the unit disk, it is convenient to choose the additive constant in such a way that $g(0)=0$. In this case, the representation $f=h+\bar{g}$ is unique and it is called the canonical representation of $f$. Such function $f$ is quasiconformal if it is absolutely continuous on lines in $\mathbf{D}$ and $\left|\omega_{f}\right|=\left|g^{\prime} / h^{\prime}\right| \leq k<1$.

We refer the reader to the books by Lehto and Virtanen [11], Ahlfors [1], and Astala, Iwaniec, and Martin [4], and to the book by Duren [7] for the general theory of quasiconformal mappings and harmonic mappings in the plane, respectively.

1.2. Quasicircles. A Jordan curve $\Gamma$ is a quasicircle if there exists a constant $M$ such that

$$
\min \left\{\operatorname{diam} \Gamma_{1}, \operatorname{diam} \Gamma_{2}\right\} \leq M\left|w_{1}-w_{2}\right|, \quad \text { for } w_{1}, w_{2} \in \Gamma,
$$

where $\Gamma_{1}$ and $\Gamma_{2}$ are the components of $\Gamma \backslash\left\{w_{1}, w_{2}\right\}$.

Ahlfors [2] proved that $\Gamma$ is a quasicircle if and only if there exists a real positive constant $C$ such that for all $w_{1}, w_{2}, w_{3}, w_{4} \in \Gamma$,

$$
\left|\left(w_{1}, w_{2}, w_{3}, w_{4}\right)\right| \leq C
$$


where $\left(w_{1}, w_{2}, w_{3}, w_{4}\right)$ is the cross ratio defined by

$$
\left(w_{1}, w_{2}, w_{3}, w_{4}\right)=\frac{w_{1}-w_{3}}{w_{1}-w_{4}} \cdot \frac{w_{2}-w_{4}}{w_{2}-w_{3}} .
$$

A quasidisk is a domain bounded by a quasicircle. In the case when $f$ is a quasiconformal function in the unit disk, $f(\mathbf{D})$ is a quasidisk if and only if $f$ has a quasiconformal extension to $\mathbf{C}$ (see [2] or [11, Theorem 8.3 on p. 98]).

1.3. Pre-Schwarzian derivatives of harmonic mappings. Let $f=h+\bar{g}$ be a locally univalent harmonic mapping in the unit disk with second complex dilatation $\omega=g^{\prime} / h^{\prime}$. The pre-Schwarzian derivative $P_{f}$ of $f$ is defined in $\mathbf{D}$ by

$$
P_{f}=\frac{\partial}{\partial z} \log \left|J_{f}\right|
$$

where $J_{f}=\left|h^{\prime}\right|^{2}-\left|g^{\prime}\right|^{2}$ is the Jacobian of the function $f$.

Notice that if $f$ is analytic (this is, if $\omega \equiv 0$ ), then $P_{f}$ coincides with the classical definition of pre-Schwarzian derivative of analytic mappings. It is also easy to check that $P_{f}=P_{\bar{f}}$. Hence, there is no loss of generality if we assume that $f=h+\bar{g}$ is sense-preserving. In this case, the pre-Schwarzian derivative of the function $f=h+\bar{g}$ equals

$$
P_{f}=\frac{\partial}{\partial z} \log \left(\left|h^{\prime}\right|^{2} \cdot\left(1-|\omega|^{2}\right)\right)=\frac{h^{\prime \prime}}{h^{\prime}}-\frac{\bar{\omega} \omega^{\prime}}{\left(1-|\omega|^{2}\right)} .
$$

A straightforward computation shows that given any $a \in \mathbf{D}$ and any sensepreserving harmonic mapping $f$ in the unit disk with canonical decomposition $f=$ $h+\bar{g}$, the function $f_{a}=f+a \bar{f}$ is also a sense-preserving harmonic mapping. Let $f_{a}=$ $h_{a}+\overline{g_{a}}$ be the canonical decomposition of $f_{a}$ and $\omega_{a}$ its second complex dilatation. Then,

$$
h_{a}=h+a g, \quad g_{a}=g+\bar{a} h, \quad \text { and } \quad \omega_{a}=\varphi_{a} \circ \omega,
$$

where $\varphi_{a}$ is the automorphism of the unit disk defined by

$$
\varphi_{a}(z)=\frac{\bar{a}+z}{1+a z}, \quad z \in \mathbf{D} .
$$

The Jacobian $J_{f_{a}}$ of $f_{a}$ equals $\left(1-|a|^{2}\right) J_{f}$. Hence, $P_{f}=P_{f_{a}}$.

As in the classical (analytic) case, the Schwarzian derivative $S_{f}$ of $f$ is

$$
S_{f}=\frac{\partial}{\partial z} P_{f}-\frac{1}{2}\left(P_{f}\right)^{2}
$$

The reader can find the main properties and several results related to the preSchwarzian and Schwarzian derivatives of locally univalent harmonic mappings defined in this section in [9].

1.4. Hyperbolic derivative of self-maps of the unit disk. Let $\omega$ be a self-map of the unit disk, this is, an analytic function in $\mathbf{D}$ with $\omega(\mathbf{D}) \subset \mathbf{D}$. As we mentioned before, the hyperbolic derivative of such a function $\omega$ is

$$
\omega^{*}(z)=\frac{\omega^{\prime}(z)\left(1-|z|^{2}\right)}{1-|\omega(z)|^{2}}, \quad z \in \mathbf{D} .
$$

Using the Schwarz-Pick lemma, we see that $\left|\omega^{*}\right| \leq 1$ in $\mathbf{D}$ and that if there exists $z_{0} \in \mathbf{D}$ with $\left|\omega^{*}\left(z_{0}\right)\right|=1$, then $\omega$ maps the unit disk conformally onto itself and $\left|\omega^{*}\right| \equiv 1$ in $\mathbf{D}$. 
Given two self-maps $\omega$ and $\varphi$ of the unit disk, the chain rule for the hyperbolic derivative holds:

$$
(\varphi \circ \omega)^{*}(z)=\varphi^{*}(\omega(z)) \cdot \omega^{*}(z) .
$$

In particular, if $\varphi$ is a conformal self-map of $\mathbf{D}$ onto $\mathbf{D}$, then $\left|(\varphi \circ \omega)^{*}\right| \equiv\left|\omega^{*}\right|$ in the unit disk.

The exact value of the upper bound of the modulus of hyperbolic derivative of the so-called lens-maps $\ell_{\alpha}$ will be of particular importance for our purposes. For $0<\alpha<1$, the mapping $\ell_{\alpha}$ is the self-map of the unit disk defined by

$$
\ell_{\alpha}(z)=\frac{\ell(z)^{\alpha}-1}{\ell(z)^{\alpha}+1}
$$

where $\ell(z)=(1+z) /(1-z)$. Note that $\left\|\ell_{\alpha}\right\|_{\infty}=1$ for all $0<\alpha<1$. In [10], it is explicitly checked that

$$
\sup _{z \in \mathbf{D}}\left|\ell_{\alpha}^{*}(z)\right|=\alpha
$$

\section{Proof of Theorem 1}

As we mentioned in Section 1.3, we have that for all $a \in \mathbf{D}, P_{f_{a}}=P_{f}$, where $f_{a}=f+a \bar{f}$. It is easy to check that $f_{a}=h_{a}+\overline{g_{a}}$ with $h_{a}=h+a g$ and $g_{a}=g+\bar{a} h$. The second complex dilatation $\omega_{a}$ of $f_{a}$ equals $\omega_{a}=\varphi_{a} \circ \omega\left(\varphi_{a}\right.$ being the automorphism of the unit disk defined by (9)). Hence, by the chain rule for the hyperbolic derivative, we get $\left|\omega_{a}^{*}\right|=\left|\omega^{*}\right|$ in the unit disk, where $\omega_{a}^{*}$ and $\omega^{*}$ are the hyperbolic derivatives of $\omega_{a}$ and $\omega$, respectively, defined by (10).

2.1. Step 1. We first prove that for all $a \in \overline{\mathbf{D}}$, the functions $h_{a}=h+a g$ have a continuous and injective extension to the whole complex plane. To do so, we bear in mind the last paragraph and use (4) to obtain that for all $z \in \mathbf{D}$,

$$
\begin{aligned}
k & \geq\left|P_{f_{a}}(z)\left(1-|z|^{2}\right)\right|+\left|\omega_{a}^{*}(z)\right| \\
& =\left|\frac{h^{\prime \prime}(z)+a g^{\prime \prime}(z)}{h^{\prime}(z)+a g^{\prime}(z)}-\frac{\overline{\omega_{a}(z)} \omega_{a}^{\prime}(z)}{1-\left|\omega_{a}(z)\right|^{2}}\right|\left(1-|z|^{2}\right)+\left|\omega_{a}^{*}(z)\right| .
\end{aligned}
$$

Making use of the triangle inequality, we get from (13):

$$
\begin{aligned}
\left|\frac{h_{a}^{\prime \prime}(z)}{h_{a}^{\prime}(z)}\left(1-|z|^{2}\right)\right| \leq & \left|\frac{h^{\prime \prime}(z)+a g^{\prime \prime}(z)}{h^{\prime}(z)+a g^{\prime}(z)}-\frac{\overline{\omega_{a}(z)} \omega_{a}^{\prime}(z)}{1-\left|\omega_{a}(z)\right|^{2}}\right|\left(1-|z|^{2}\right) \\
& +\left|\frac{\overline{\omega_{a}(z)} \omega_{a}^{\prime}(z)}{1-\left|\omega_{a}(z)\right|^{2}}\right|\left(1-|z|^{2}\right) \\
\leq & \left|P_{f_{a}}(z)\left(1-|z|^{2}\right)\right|+\left|\omega_{a}^{*}(z)\right| \leq k,
\end{aligned}
$$

which implies, by the classical result due to Becker for analytic functions, that for each $a \in \mathbf{D}$, the function $h_{a}$ is univalent and can be extended to a continuous and injective mapping $\widetilde{h}_{a}$ in the closed unit disk. Moreover (see [3]), the function

$$
H_{a}(z)= \begin{cases}\widetilde{h}_{a}(z), & |z| \leq 1 \\ h_{a}\left(\frac{1}{\bar{z}}\right)+u_{a}\left(\frac{1}{\bar{z}}\right), & |z|>1\end{cases}
$$


(where, for $\left.z \in \mathbf{D} \backslash\{0\}, u_{a}(z)=h_{a}^{\prime}(z)\left(1-|z|^{2}\right) / \bar{z}\right)$ is $K$-quasiconformal in the complex plane with $K=(1+k) /(1-k)$. Hence, in particular, $H_{a}$ is continuous and injective in $\mathbf{C}$.

Take a complex number $\lambda=e^{i \theta} \in \partial \mathbf{D}$ and define

$$
H_{\lambda}(z)=\lim _{r \rightarrow 1^{-}} H_{r e^{i \theta}}(z), \quad z \in \mathbf{C} .
$$

According to [11, Theorem 5.3], the functions $H_{\lambda}$ are either constant functions, functions from $\mathbf{C}$ onto two points, or $K$-quasiconformal functions in the complex plane. But using Hurwitz's theorem, we see that for each $\lambda \in \partial \mathbf{D}$, the function $h+\lambda g$ is either a constant or univalent in $\mathbf{D}$. Such a function $h+\lambda g$ cannot be constant for any $|\lambda|=1$ because it would imply that $\left|h^{\prime}\right| \equiv\left|g^{\prime}\right|$ in the unit disk and this is a contradiction with the condition $\left|g^{\prime}(0)\right|<\left|h^{\prime}(0)\right|$ that holds for any sensepreserving harmonic mapping. Therefore, we conclude that $h+\lambda g$ are univalent for all $\lambda \in \partial \mathbf{D}$. Since $H_{\lambda}$ coincides with $h+\lambda g$ in $\mathbf{D}$, we obtain that $H_{\lambda}$ is a $K$ quasiconformal function in the complex plane for each $|\lambda|=1$. Thus $H_{\lambda}$ is continuous and one-to-one in $\mathbf{C}$ for all $|\lambda|=1$ (hence for all $|\lambda| \leq 1$ ).

2.2. Step 2. The next step is to construct an explicit candidate for a continuous and injective extension of $f$ to the whole complex plane.

We define, for each $z \in \mathbf{C}$, the function

$$
G(z)=H_{1}(z)-H(z)
$$

(where $H=H_{0}$ and $H_{1}$ denote the corresponding extension of $h_{0}=h$ and $h_{1}=h+g$, respectively, as in (14)), to obtain a continuous extension $G$ of $g$ to $\mathbf{C}$ given by

$$
G(z)= \begin{cases}g(z), & |z|<1 \\ \widetilde{g}(z)=\widetilde{h}_{1}(z)-\widetilde{h}(z), & |z|=1 \\ g\left(\frac{1}{\bar{z}}\right)+v\left(\frac{1}{\bar{z}}\right), & |z|>1\end{cases}
$$

where

$$
v(z)=\frac{g^{\prime}(z)\left(1-|z|^{2}\right)}{\bar{z}}, \quad z \in \mathbf{D} \backslash\{0\} .
$$

Note that for each $z \in \mathbf{C}$ and all $a \in \overline{\mathbf{D}}$,

$$
H_{a}(z)=H(z)+a G(z) .
$$

Hence, since $H_{a}$ are univalent in the complex plane for all $a \in \overline{\mathbf{D}}$, we see that $H+a G$ is injective in $\mathbf{C}$ for all such $a$.

We now construct the function defined by (5):

$$
F(z)=H(z)+\overline{G(z)}= \begin{cases}f(z), & |z|<1 \\ \widetilde{h}(z)+\overline{\widetilde{g}(z)}, & |z|=1 \\ f\left(\frac{1}{\bar{z}}\right)+U\left(\frac{1}{\bar{z}}\right), & |z|>1\end{cases}
$$

where $U$ is defined in $\mathbf{D} \backslash\{0\}$ by

$$
U(z)=\frac{h^{\prime}(z)\left(1-|z|^{2}\right)}{\bar{z}}+\frac{\overline{g^{\prime}(z)}\left(1-|z|^{2}\right)}{z} .
$$

2.3. Step 3. We are going to show that the function $F$ as in (5) is injective in the complex plane. We use the arguments from [8]. 
Assume, in order to get a contradiction, that $F\left(z_{1}\right)=F\left(z_{2}\right)$ for two different points $z_{1}$ and $z_{2}$ in $\mathbf{C}$. Then, we have

$$
H\left(z_{1}\right)-H\left(z_{2}\right)=\overline{G\left(z_{2}\right)-G\left(z_{1}\right)} .
$$

By hypothesis, $H$ is univalent in the complex plane. Hence, $H\left(z_{1}\right) \neq H\left(z_{2}\right)$. Denote by $\theta=\arg \left\{H\left(z_{1}\right)-H\left(z_{2}\right)\right\} \in[0,2 \pi)$. Thus, we obtain from (15) that

$$
e^{-i \theta}\left(H\left(z_{1}\right)-H\left(z_{2}\right)\right)=e^{-i \theta}\left(\overline{G\left(z_{1}\right)-G\left(z_{2}\right)}\right)
$$

is a positive real number. Therefore, after taking conjugates on the right hand side of the last equality, we get $H\left(z_{1}\right)-H\left(z_{2}\right)=e^{2 i \theta}\left(G\left(z_{1}\right)-G\left(z_{2}\right)\right)$, which contradicts the fact that the function $H-e^{2 i \theta} G$ is univalent in $\mathbf{C}$.

2.4. Step 4. Finally, we will check that the function $F$ defined by (5) is a homeomorphism of $\mathbf{C}$ onto itself.

That the function $F$ is continuous in the complex plane follows from the fact that both $H$ and $G$ are continuous in C. Moreover, a straightforward computation (that mainly uses the triangle inequality and that $f$ is sense-preserving) gives $\lim _{|z| \rightarrow \infty}|F(z)|=\infty$. Hence, we can extend continuously (and in a one-to-one manner) the function $F$ to the Riemann sphere $\overline{\mathbf{C}}$ in such a way that $F(\infty)=\infty$. Therefore (see for instance [13, Theorem 5.6 on p. 167]), $F$ is a homeomorphism of the Riemann sphere onto $F(\overline{\mathbf{C}})$, so that $F(\overline{\mathbf{C}})=\overline{\mathbf{C}}$. Since $F(\infty)=\infty$, we conclude that $F$ is a homeomorphism of the complex plane onto itself.

\section{Proof of Theorem 2}

We would first like to remark that the hypotheses $\|\omega\|_{\infty}<1$ in this theorem cannot be removed. To do so, consider the sense-preserving harmonic mapping $f=$ $z+\bar{g}$, where $g^{\prime}$ equals the lens-map $\ell_{\alpha}$ defined by (11). Note that the second complex dilatation $\omega$ of $f$ equals $\ell_{\alpha}$. Then, on the one side, using (12) we obtain

$$
\sup _{z \in \mathbf{D}}\left(\left|P_{f}(z)\right|\left(1-|z|^{2}\right)+\left|\omega^{*}(z)\right|\right)=\sup _{z \in \mathbf{D}}\left(\left|\overline{\ell_{\alpha}} \ell_{\alpha}^{*}(z)\right|+\left|\ell_{\alpha}^{*}(z)\right|\right) \leq 2 \alpha .
$$

Therefore, by choosing any $\alpha<1 / 2$, we have that (4) holds (with $k=2 \alpha<1$ ). On the other side, the function $f$ is not quasiconformal since $\left\|\ell_{\alpha}\right\|_{\infty}=1$.

Going back to the proof, we first notice that it is enough to prove Theorem 2 for sense-preserving harmonic mappings $f$ that have a canonical decomposition of the form $f=h+\bar{g}$, where both $f$ and $g$ are analytic in $\overline{\mathbf{D}}$. This is because given any positive real number $0<r<1$, we can define the sense-preserving harmonic function $f_{r}(z)=f(r z)=h(r z)+\overline{g(r z)}$. Each of these functions $f_{r}$ has second complex dilatation $\omega_{r}=\omega(r z)$, so that $\left\|\omega_{r}\right\|_{\infty} \leq\|\omega\|_{\infty}<1$.

Also, it is easy to check that if $f$ satisfies (4), then

$\sup _{z \in \mathbf{D}}\left\{\left|P_{f_{r}}(z)\right|\left(1-|z|^{2}\right)+\left|\omega_{r}^{*}(z)\right|\right\} \leq r \sup _{z \in \mathbf{D}}\left|P_{f}(z)\right|\left(1-|z|^{2}\right)+r \sup _{z \in \mathbf{D}}\left|\omega^{*}(z)\right| \leq r k<k$,

so that $f_{r}$ satisfies (4) as well.

Now, if we check that $f_{r}$ can be extended to a $K$-quasiconformal function in the complex plane for some constant $K \geq 1$ that does not depend on $r$, then we can use [11, Theorem 5.3] and argue as in Subsection 2.1 to conclude that $f$ can be also extended to a $K$-quasiconformal function in $\mathbf{C}$. Hence, from now on, we can assume 
that the functions $h$ and $g$ in the canonical decomposition of $f$ are both analytic functions in $\overline{\mathbf{D}}$.

3.1. Some useful lemmas. We will use the following three lemmas.

Lemma 1. Let $f=h+\bar{g}$ be a sense-preserving harmonic mapping in $\mathbf{D}$ with complex dilatation $\omega \not \equiv 0$. Assume that $\|\omega\|_{\infty}<1$ and that $f$ satisfies (4). Then, the analytic functions $h_{a}=h+a g$ are univalent in $\mathbf{D}$ for all $0 \leq|a|<\delta$, where

$$
1<\delta=\frac{1+k\|\omega\|_{\infty}}{k+\|\omega\|_{\infty}} \leq \frac{1}{\|\omega\|_{\infty}}
$$

Moreover, $h_{a}$ has a continuous and injective extension $\widetilde{h}_{a}$ to $\overline{\mathbf{D}}$.

Proof. Notice that the fact that $h_{a}$ is univalent in the unit disk for all $|a| \leq 1$ has been already proved in Subsection 2.1. Indeed, we showed in that subsection that $h_{a}$ has a continuous and injective extension to $\overline{\mathbf{D}}$ of the form $\widetilde{h}_{a}=\widetilde{h}+a \widetilde{g}$ for suitable functions $\widetilde{h}$ and $\widetilde{g}$ that coincides with $h$ and $g$ in the unit disk, respectively. Hence, we can extend these functions $h_{a}$ for any value of $a$ in the complex plane via the formula $\widetilde{h}_{a}=\widetilde{h}+a \widetilde{g}$. Note that if we can prove that the function $h_{a}$ (for any $a \in \mathbf{C})$ can be extended continuously to the closed unit disk, then the extension will coincide with $\widetilde{h}_{a}$.

We are going to show that these functions $h_{a}$ keep the univalence property in $\mathbf{D}$ for $1<|a|<\delta$ and that the corresponding extensions $\widetilde{h}_{a}$ are injective in $\overline{\mathbf{D}}$.

Since $|a|<\delta \leq 1 /\|\omega\|_{\infty}$ and $h_{a}^{\prime}=h^{\prime}(1+a w)$, we see that $h_{a}$ is locally univalent in the unit disk. Therefore, we can compute the pre-Schwarzian derivative of $h_{a}$ to obtain

$$
\frac{h_{a}^{\prime \prime}}{h_{a}^{\prime}}=\frac{h^{\prime \prime}}{h^{\prime}}+\frac{a \omega^{\prime}}{1+a \omega}
$$

Keeping in mind Formula (8) for the pre-Schwarzian derivative of $f$ and (16), we get

$$
\begin{aligned}
\frac{h_{a}^{\prime \prime}}{h_{a}^{\prime}} & =P_{f}+\omega^{\prime}\left(\frac{\bar{\omega}}{1-|\omega|^{2}}+\frac{a}{1+a \omega}\right) \\
& =P_{f}+\frac{\omega^{\prime}}{1-|\omega|^{2}}\left(\bar{\omega}+a \frac{1-|\omega|^{2}}{1+a \omega}\right)=P_{f}+\frac{\omega^{\prime}}{1-|\omega|^{2}} \cdot \frac{\bar{\omega}+a}{1+a \omega},
\end{aligned}
$$

which implies that for all $z \in \mathbf{D}$,

$$
\left|\frac{h_{a}^{\prime \prime}(z)}{h_{a}^{\prime}(z)}\right|\left(1-|z|^{2}\right) \leq\left|P_{f}(z)\right|\left(1-|z|^{2}\right)+\left|\omega^{*}(z)\right| \cdot\left|\frac{\omega(z)+\bar{a}}{1+a \omega(z)}\right| .
$$

Now, note that since $f$ is supposed to satisfy (4), we get that its second complex dilatation $\omega$ satisfies $\left|\omega^{*}\right| \leq k$ in the unit disk. Moreover, we also have for all $|z|<1$,

$$
\left|P_{f}(z)\right|\left(1-|z|^{2}\right) \leq k-\left|\omega^{*}(z)\right| .
$$

Then, we obtain from (17):

$$
\left|\frac{h_{a}^{\prime \prime}(z)}{h_{a}^{\prime}(z)}\right|\left(1-|z|^{2}\right) \leq k+\left|\omega^{*}(z)\right|\left(\left|\frac{\omega(z)+\bar{a}}{1+a \omega(z)}\right|-1\right) \leq k\left|\frac{\omega(z)+\bar{a}}{1+a \omega(z)}\right| .
$$


In view of the inequality (18), the proof of the lemma will follow from the classical Becker's criterion for analytic functions once we show that

$$
\sup _{z \in \mathbf{D}}\left|\frac{\omega(z)+\bar{a}}{1+a \omega(z)}\right|<\frac{1}{k}
$$

for all $1<|a|<\delta$. To check that (19) holds, we first note that for $\lambda=a /|a|$,

$$
\left|\frac{\omega(z)+\bar{a}}{1+a \omega(z)}\right|=\left|\frac{\lambda \omega(z)+\lambda \bar{a}}{1+\bar{\lambda} a \lambda \omega(z)}\right|=\left|\frac{\lambda \omega(z)+|a|}{1+|a| \lambda \omega(z)}\right| \text {. }
$$

Therefore,

$$
\sup _{z \in \mathbf{D}}\left|\frac{\lambda \omega(z)+\lambda \bar{a}}{1+\bar{\lambda} a \lambda \omega(z)}\right|=\sup _{z \in \mathbf{D}}\left|\frac{\lambda \omega(z)+|a|}{1+|a| \lambda \omega(z)}\right| \leq \sup _{|z| \leq\|\omega\|_{\infty}}\left|\frac{z+|a|}{1+|a| z \mid}\right| .
$$

Now, using again that $|a|<\delta \leq 1 /\|\omega\|_{\infty}$, we have that the linear fractional transformation

$$
T(z)=\frac{z+|a|}{1+|a| z}
$$

maps the disk $D\left(0,\|\omega\|_{\infty}\right)$ of center at $z=0$ and radius $\|\omega\|_{\infty}$ onto a disk $D$, say. Moreover, the Taylor coefficients of $T$ are real numbers, hence $D$ is symmetric with respect to the real axes. A straightforward calculation shows that the Möbius transformation $T$ maps the interval $\left(-\|\omega\|_{\infty},\|\omega\|_{\infty}\right)$ onto the interval

$$
\left(\frac{|a|+\|\omega\|_{\infty}}{1+|a|\|\omega\|_{\infty}}, \frac{|a|-\|\omega\|_{\infty}}{1-|a|\|\omega\|_{\infty}}\right)
$$

so that

$$
\sup _{|z| \leq\|\omega\|_{\infty}}\left|\frac{z+|a|}{1+|a| z \mid}\right|=\frac{|a|-\|\omega\|_{\infty}}{1-|a|\|\omega\|_{\infty}}<\frac{1}{k}
$$

since

$$
|a|<\delta=\frac{1+k\|\omega\|_{\infty}}{k+\|\omega\|_{\infty}}
$$

Thus (19) holds, which completes the proof.

Lemma 2. Let $f=h+\bar{g}$ satisfy the hypothesis of Lemma 1. Assume in addition that both $h$ and $g$ are analytic functions in $\overline{\mathbf{D}}$. Then,

$$
\sup _{\alpha, \beta \in \overline{\mathbf{D}}, \alpha \neq \beta}\left|\frac{g(\alpha)-g(\beta)}{h(\alpha)-h(\beta)}\right| \leq \frac{1}{\delta}=\frac{k+\|\omega\|_{\infty}}{1+k\|\omega\|_{\infty}}<1 .
$$

Proof. Fix an arbitrary point $\beta \in \overline{\mathbf{D}}$ and define the function

$$
\psi_{\beta}(\alpha)=\left\{\begin{array}{ll}
\frac{g(\alpha)-g(\beta)}{h(\alpha)-h(\beta)}, & \alpha \neq \beta, \\
\omega(\alpha), & \alpha=\beta,
\end{array} \quad \alpha \in \overline{\mathbf{D}},\right.
$$

where $\omega$ is the second complex dilatation of $f$.

The function $\psi_{\beta}$ is continuous in $|\alpha| \leq 1$, so that there exists $\alpha_{0} \in \overline{\mathbf{D}}$ such that $S=\sup _{\alpha \in \overline{\mathbf{D}}}\left|\psi_{\beta}(\alpha)\right|=\left|\psi_{\beta}\left(\alpha_{0}\right)\right|$.

If $\alpha_{0}=\beta$, then $S \leq\|\omega\|_{\infty}$ and the result follows because $\delta \leq 1 /\|\omega\|_{\infty}$. 
Assume that $\alpha_{0} \neq \beta$. In order to get a contradiction, suppose that $S>1 / \delta$. Then, there exist a positive real number $\varepsilon<\delta,|\lambda|=1$, and $\alpha_{1} \in \overline{\mathbf{D}}$ with

$$
\frac{g\left(\alpha_{1}\right)-g(\beta)}{h\left(\alpha_{1}\right)-h(\beta)}=\frac{\lambda}{\delta-\varepsilon}
$$

which implies that the function $h-\bar{\lambda}(\delta-\varepsilon) g$ is not univalent in $\overline{\mathbf{D}}$. This is in contradiction with the result obtained in Lemma 1.

The third lemma is related to the analytic part $h$ of $f$.

Lemma 3. Let $f=h+\bar{g}$ ba a sense-preserving harmonic mapping in the unit disk for which (4) holds. For any positive real number $r<1$, define the function $h_{r}$ by $h_{r}(z)=h(r z), z \in \mathbf{D}$. Then, there exists a constant $M>0$ such that for all $r \in(0,1]$ and all four different points $w_{i}, i=1,2,3,4$, in $\Gamma_{r}=h_{r}(\partial \mathbf{D})$, the modulus of the cross ratio $\left(w_{1}, w_{2}, w_{3}, w_{4}\right)$ defined by $(7)$ is bounded by $M$.

Proof. Using the arguments in the proof of Theorem 1, since we are assuming that $f$ satisfies (4), we have that the analytic part $h$ of $f$ can be extended continuously to an injective function $\widetilde{h}$ in $\overline{\mathbf{D}}$ with the property that $\widetilde{h}(\partial \mathbf{D})$ is a quasicircle.

Hence, given any four different points $w_{i}, i=1,2,3,4$, in $\Gamma=\widetilde{h}(\partial \mathbf{D})$, the modulus of the cross ratio $\left|\left(w_{1}, w_{2}, w_{3}, w_{4}\right)\right|$ is bounded by a constant $M$. This implies that for any four different points $\left|\zeta_{i}\right|=1, i=1,2,3,4$, we have

$$
\left|\frac{\widetilde{h}\left(\zeta_{1}\right)-\widetilde{h}\left(\zeta_{3}\right)}{\widetilde{h}\left(\zeta_{1}\right)-\widetilde{h}\left(\zeta_{4}\right)} \cdot \frac{\widetilde{h}\left(\zeta_{2}\right)-\widetilde{h}\left(\zeta_{4}\right)}{\widetilde{h}\left(\zeta_{2}\right)-\widetilde{h}\left(\zeta_{3}\right)}\right| \leq M .
$$

Now, if we fix four different points $\alpha_{i}, i=1,2,3,4$, on the boundary of the unit disk and define the function

$$
\hbar(\lambda)=\frac{\widetilde{h}\left(\lambda \alpha_{1}\right)-\widetilde{h}\left(\lambda \alpha_{3}\right)}{\widetilde{h}\left(\lambda \alpha_{1}\right)-\widetilde{h}\left(\lambda \alpha_{4}\right)} \cdot \frac{\widetilde{h}\left(\lambda \alpha_{2}\right)-\widetilde{h}\left(\lambda \alpha_{4}\right)}{\widetilde{h}\left(\lambda \alpha_{2}\right)-\widetilde{h}\left(\lambda \alpha_{3}\right)}, \quad \lambda \in \overline{\mathbf{D}},
$$

we conclude (using (20) and the Maximum Modulus Principle) that for all $0<r<1$, the inequality

$$
\left|\frac{h\left(r \alpha_{1}\right)-h\left(r \alpha_{3}\right)}{h\left(r \alpha_{1}\right)-h\left(r \alpha_{4}\right)} \cdot \frac{h\left(r \alpha_{2}\right)-h\left(r \alpha_{4}\right)}{h\left(r \alpha_{2}\right)-h\left(r \alpha_{3}\right)}\right| \leq M
$$

holds. Since $\alpha_{i}, i=1,2,3,4$ were arbitrary and since $w \in h_{r}(\partial \mathbf{D})$ if and only if $w=h(r \alpha)$ for some $\alpha \in \partial \mathbf{D}$, the conclusion of the lemma follows from (21).

We now have all the tools to prove Theorem 2 .

The proof of Theorem 2. Note that if $\omega \equiv 0$ in the unit disk, Theorem 2 holds by the classical theorems due to Becker and Ahlfors that we have mentioned in the introduction. Therefore, we will assume that $\omega \neq \equiv 0$ in $\mathbf{D}$.

We first show that if

$$
k<\frac{1-\|\omega\|_{\infty}}{1+\|\omega\|_{\infty}}
$$

then the function $F$ defined by (5) is $K$-quasiconformal in the whole complex plane, where $K$ is the constant defined by (6). To do so, it suffices to prove (since $h$ satisfies (2)) that for all $|z| \neq 1$, the dilatation $\mu_{F}=F_{\bar{z}} / F_{z}$ of $F$ is bounded by $(K-1) /(K+1)$. (Notice that this is enough for our purposes since $|z|=1$ is a removable set in $\mathbf{C}$.) 
If $|z|<1$, then $\left|\mu_{F}(z)\right|=|\omega(z)| \leq\|\omega\|_{\infty}$.

Let now $w$ have modulus bigger than 1 . Hence, $w=1 / \bar{z}$ for some $|z|<1$ and a straightforward computation shows that

$$
\begin{aligned}
\left|\mu_{F}(w)\right| & =\left|\frac{h^{\prime}(z)+U_{z}(z)}{\overline{g^{\prime}(z)}+U_{\bar{z}}(z)}\right|=\left|\frac{z^{2} h^{\prime \prime}(z)\left(1-|z|^{2}\right)-\overline{z g^{\prime}(z)}}{z^{2} g^{\prime \prime}(z)\left(1-|z|^{2}\right)-\overline{z h^{\prime}(z)}}\right| \\
& =\left|\frac{z^{2} \frac{h^{\prime \prime}(z)}{h^{\prime}(z)}\left(1-|z|^{2}\right)-\overline{z \omega(z)} \frac{\overline{h^{\prime}(z)}}{h^{\prime}(z)}}{z^{2} \frac{g^{\prime \prime}(z)}{h^{\prime}(z)}\left(1-|z|^{2}\right)-\bar{z} \frac{\overline{h^{\prime}(z)}}{h^{\prime}(z)}}\right| \leq \frac{\left|\frac{h^{\prime \prime}(z)}{h^{\prime}(z)}\right|\left(1-|z|^{2}\right)+\|\omega(z)\|_{\infty}}{1-\left|\frac{g^{\prime \prime}(z)}{h^{\prime}(z)}\right|\left(1-|z|^{2}\right)} .
\end{aligned}
$$

On the one hand, using that $f$ satisfies (4), we get

$$
\left|\frac{h^{\prime \prime}(z)}{h^{\prime}(z)}-\frac{\overline{\omega(z)} \omega^{\prime}(z)}{1-|\omega(z)|^{2}}\right|\left(1-|z|^{2}\right) \leq k-\left|\omega^{*}(z)\right|,
$$

which implies (by the triangle inequality)

$$
\left|\frac{h^{\prime \prime}(z)}{h^{\prime}(z)}\left(1-|z|^{2}\right)\right| \leq k-\left|\omega^{*}(z)\right|+\left|\omega(z) \omega^{*}(z)\right| \leq k-\left(1-\|\omega\|_{\infty}\right)\left|\omega^{*}(z)\right| .
$$

On the other hand, we have $g^{\prime \prime}=\left(\omega h^{\prime}\right)^{\prime}=\omega^{\prime} h^{\prime}+\omega h^{\prime \prime}$ in the unit disk. Hence, for all $z \in \mathbf{D}$ we obtain

$$
\begin{aligned}
\frac{g^{\prime \prime}(z)}{h^{\prime}(z)}\left(1-|z|^{2}\right) & =\left(\omega(z) \frac{h^{\prime \prime}(z)}{h^{\prime}(z)}+\omega^{\prime}(z)\right)\left(1-|z|^{2}\right) \\
& =\omega(z) \frac{h^{\prime \prime}(z)}{h^{\prime}(z)}\left(1-|z|^{2}\right)+\frac{\omega^{\prime}(z)\left(1-|z|^{2}\right)}{1-|\omega(z)|^{2}}\left(1-|\omega(z)|^{2}\right) \\
& =\omega(z) P_{f}(z)\left(1-|z|^{2}\right)+\frac{\omega^{\prime}(z)\left(1-|z|^{2}\right)}{1-|\omega(z)|^{2}} .
\end{aligned}
$$

Therefore,

$$
\begin{aligned}
\left|\frac{g^{\prime \prime}(z)}{h^{\prime}(z)}\right|\left(1-|z|^{2}\right) & \leq|\omega(z)|\left|P_{f}(z)\right|\left(1-|z|^{2}\right)+\left|\omega^{*}(z)\right| \\
& \leq\|\omega\|_{\infty}\left(k-\left|\omega^{*}(z)\right|\right)+\left|\omega^{*}(z)\right| \\
& =k\|\omega\|_{\infty}+\left(1-\|\omega\|_{\infty}\right)\left|\omega^{*}(z)\right| .
\end{aligned}
$$

Using (24) and (25) in (23), we deduce

$$
\begin{aligned}
\left|\mu_{F}(w)\right| & \leq \frac{k-\left(1-\|\omega\|_{\infty}\right)\left|\omega^{*}(z)\right|+\|\omega\|_{\infty}}{1-\left(k\|\omega\|_{\infty}+\left(1-\|\omega\|_{\infty}\right)\left|\omega^{*}(z)\right|\right)} \\
& =\frac{k+\|\omega\|_{\infty}-\left(1-\|\omega\|_{\infty}\right)\left|\omega^{*}(z)\right|}{1-k\|\omega\|_{\infty}-\left(1-\|\omega\|_{\infty}\right)\left|\omega^{*}(z)\right|} .
\end{aligned}
$$

Define the function $\rho(x):[0, k] \rightarrow \mathbf{R}$ by

$$
\rho(x)=\frac{k+\|\omega\|_{\infty}-\left(1-\|\omega\|_{\infty}\right) x}{1-k\|\omega\|_{\infty}-\left(1-\|\omega\|_{\infty}\right) x} .
$$


Bearing in mind that $(22)$ holds, we see that $\rho^{\prime}(x)<0$ for all $x \in[0, k]$. Thus,

$$
\rho(x) \leq \rho(0)=\frac{k+\|\omega\|_{\infty}}{1-k\|\omega\|_{\infty}}=\frac{K-1}{K+1} .
$$

We then conclude by (26) that for all $|w|>1$ the inequality $\left|\mu_{F}(w)\right| \leq(K-1) /(K+1)$ holds. A straightforward computation shows that $\|\omega\|_{\infty} \leq(K-1) /(K+1)$ as well. Therefore, we obtain, in fact, $\left|\mu_{F}(z)\right| \leq(K-1) /(K+1)$ for all $|z| \neq 1$. This proves that the function $F$ defined by $(5)$ is $K$-quasiconformal whenever (22) holds.

To finish the proof of Theorem 2, we are to show that even if (22) does not hold (so that we cannot ensure that the function defined by (5) is quasiconformal), the harmonic mapping $f$ can be extended to some quasiconformal map in the complex plane.

By considering the dilations $f_{r}$ defined by $f_{r}(z)=f(r z)$ and using [11, Theorem 5.3] one more time, we have that the result will follow once we prove that it holds under the additional assumption that $f$ has a canonical decomposition of the form $h+\bar{g}$, where $h$ and $g$ are analytic functions in $\overline{\mathbf{D}}$, provided that we can show that each of the functions $f_{r}$ has a $K$-quasiconformal extension $F_{r}$ to $\mathbf{C}$, where $K$ is a constant that does not depend on $r$ (and is not necessarily equal to that constant $K$ defined by (6)). To do so, we are going to check that the cross ratio of any four different points in $\Gamma_{r}=f_{r}(\partial \mathbf{D})$ is uniformly bounded by a universal positive constant $C$ for all $r \in(0,1)$. This will be enough for our purposes (see [2]) since, in this case, we conclude that $\Gamma_{r}$ is a quasicircle so that $f_{r}$ admits a $K$-quasiconformal reflection, where the constant $K$ only depends on $C$ (hence, $K$ is independent of $r$, as needed).

Note that, at this point, we know that for each $\lambda \in \overline{\mathbf{D}}$, the analytic function $\varphi_{r, \lambda}=h_{r}+\lambda g_{r}$ has a $K$-quasiconformal extension $\Phi_{r, \lambda}$ to $\mathbf{C}$. Hence, the Jordan curve $\Phi_{r, \lambda}(\partial \mathbf{D})$ is a quasicircle for each $|\lambda| \leq 1$. As a consequence, we see that given any four different arbitrary points $w_{i} \in \Phi_{r, \lambda}(\partial \mathbf{D}), i=1,2,3,4$, the cross ratio $\left(w_{1}, w_{2}, w_{3}, w_{4}\right)$ defined by $(7)$ is bounded by a constant $K_{r, \lambda}$ (that can depend on $r$ and on $\lambda)$.

Take four different arbitrary points $w_{i}, i=1,2,3,4$ in $\Gamma_{r}=f_{r}(\partial \mathbf{D})$. The fact that $w_{i} \in \Gamma_{r}$ implies that there exists $\left|\zeta_{i}\right|=1$ such that $w_{i}=f_{r}\left(\zeta_{i}\right)=h_{r}\left(\zeta_{i}\right)+\overline{g_{r}\left(\zeta_{i}\right)}=$ $h_{i}+\overline{g_{i}}, i=1,2,3,4$. Thus, we can write

$$
\begin{aligned}
\left|\left(w_{1}, w_{2}, w_{3}, w_{4}\right)\right| & =\left|\frac{h_{1}-h_{3}+\overline{g_{1}-g_{3}}}{h_{1}-h_{4}+\overline{g_{1}-g_{4}}} \cdot \frac{h_{2}-h_{4}+\overline{g_{2}-g_{4}}}{h_{2}-h_{3}+\overline{g_{2}-g_{3}}}\right| \\
& =\left|\frac{h_{1}-h_{3}+\lambda_{13}\left(g_{1}-g_{3}\right)}{h_{1}-h_{4}+\lambda_{14}\left(g_{1}-g_{4}\right)}\right| \cdot\left|\frac{h_{2}-h_{4}+\lambda_{24}\left(g_{2}-g_{4}\right)}{h_{2}-h_{3}+\lambda_{23}\left(g_{2}-g_{3}\right)}\right| \\
& =\left|\frac{h_{13}}{h_{14}} \cdot \frac{h_{24}}{h_{23}}\right| \cdot\left|\frac{1+\lambda_{13} \Lambda_{13}}{1+\lambda_{14} \Lambda_{14}}\right| \cdot\left|\frac{1+\lambda_{24} \Lambda_{24}}{1+\lambda_{23} \Lambda_{23}}\right|,
\end{aligned}
$$

where $\lambda_{i j}\left(g_{i}-g_{j}\right)=\overline{g_{i}-g_{j}}\left(\right.$ so that $\left.\left|\lambda_{i j}\right|=1\right), h_{i j}=h_{i}-h_{j}$, and $\Lambda_{i j}=\left(g_{i}-g_{j}\right) /\left(h_{i}-\right.$ $\left.h_{j}\right)$, with $i, j=1,2,3,4$.

Using Lemma 3, we see that there exists a constant $M$ that does not depend on $r$ such that for any four different points $\left|\zeta_{i}\right|=1, i=1,2,3,4$,

$$
\left|\left(h_{r}\left(\zeta_{1}\right), h_{r}\left(\zeta_{2}\right), h_{r}\left(\zeta_{3}\right), h_{r}\left(\zeta_{4}\right)\right)\right|=\left|\frac{h_{13}}{h_{14}} \cdot \frac{h_{24}}{h_{23}}\right| \leq M
$$


Hence, we deduce that the first factor in (27) is uniformly bounded by that constant $M$.

On the other hand, define

$$
\delta_{r}=\frac{1+k\left\|\omega_{r}\right\|_{\infty}}{k+\left\|\omega_{r}\right\|_{\infty}}
$$

(where $\omega_{r}$ is the dilatation of $f_{r}$ ) and use Lemma 2 to obtain

$$
\begin{aligned}
\left|\frac{1+\lambda_{13} \Lambda_{13}}{1+\lambda_{14} \Lambda_{14}}\right| \cdot\left|\frac{1+\lambda_{24} \Lambda_{24}}{1+\lambda_{23} \Lambda_{23}}\right| & \leq\left(\frac{1+1 / \delta_{r}}{1-1 / \delta_{r}}\right)^{2} \\
& =\left(\frac{1+k}{1-k}\right)^{2} \cdot\left(\frac{1+\left\|\omega_{r}\right\|_{\infty}}{1-\left\|\omega_{r}\right\|_{\infty}}\right)^{2} \\
& \leq\left(\frac{1+k}{1-k}\right)^{2} \cdot\left(\frac{1+\|\omega\|_{\infty}}{1-\|\omega\|_{\infty}}\right)^{2} .
\end{aligned}
$$

Therefore, as a direct consequence of (28) and (29), we get from (27)

$$
\left|\left(w_{1}, w_{2}, w_{3}, w_{4}\right)\right| \leq M \cdot\left(\frac{1+k}{1-k}\right)^{2} \cdot\left(\frac{1+\|\omega\|_{\infty}}{1-\|\omega\|_{\infty}}\right)^{2}=C .
$$

This proves that $f_{r}$ (hence $f$ ) admits a $K$-quasiconformal reflection, so that $f$ has a quasiconformal extension to C. This ends the proof of Theorem 2.

Acknowledgements. We are indebted to Professor Martin Chuaqui for proposing us to study the problem that we have treated in this paper. Also, for the encouragement and guidance that he provides us with at every moment.

We want to thank Professor Daniel Faraco as well for his interest and several stimulating conversations on the subject.

Part of this work was developed during the first author's stay at the Universidad Autónoma de Madrid as a Visiting Professor. He would like to thank the Department of Mathematics for its hospitality.

\section{References}

[1] Ahlfors, L.: Lectures on quasiconformal mappings. - Van Nostrand, Princeton, NJ, 1966.

[2] Ahlfors, L.: Quasiconformal reflections. - Acta Math. 109, 1963, 291-301.

[3] Ahlfors, L.: Sufficient conditions for quasiconformal extension. - Ann. of Math. Stud. 79, 1974, 23-29.

[4] Astala, K., T. Iwaniec, and G. Martin: Elliptic partial differential equations and quasiconformal mappings in the plane. - Princeton Univ. Press, Princeton and Oxford, 2009.

[5] BECKER, J.: Löwnersche differentialgleichung und quasikonform fortsetzbare schlichte functionen. - J. Reine Angew. Math. 255, 1972, 23-43.

[6] Becker, J., and Ch. Pommerenke: Schlichtheitskriterien und Jordangebiete. - J. Reine Angew. Math. 354, 1984, 74-94.

[7] Duren, P.: Harmonic mappings in the plane. - Cambridge Univ. Press, Cambridge, 2004.

[8] Hernández, R., and M. J. Martín: Stable geometric properties of analytic and harmonic functions. - Preprint, available from http://www.uam.es/mariaj.martin.

[9] Hernández, R., and M. J. Martín: Pre-Schwarzian and Schwarzian derivatives of harmonic mappings. - Preprint, available from http://www.uam.es/mariaj.martin. 
[10] Hosokawa, T., and S. Ohno: Topological structures of the set of composition operators on the Bloch space. - J. Math. Anal. Appl. 314, 2006, 736-748.

[11] Lehto, O., and K. I. Virtanen: Quasiconformal mappings in the plane. - Springer-Verlag, Berlin, Heidelberg, New York, 1973.

[12] Lewy, H.: On the non-vanishing of the jacobian in certain one-to-one mappings. - Bull. Amer. Math. Soc. 42, 1936, 689-692.

[13] Munkres, J. R.: Topology, a first course. - Prentice-Hall, Inc., Englewood Cliffs, New Jersey, 1975.

Received 26 June 2012 • Accepted 2 November 2012 